\title{
ESTIMATIVAS DA VAZÃO MÁXIMA E CAPACIDADE DE SATURAÇÃO HÍDRICA DA BACIA HIDROGRÁFICA DO ALTO DO RIO LIGEIRO EM PATO BRANCO, PR
}

\author{
Andrea Sartori Jabur ${ }^{1}$, Nivaldo Eduardo Rizzi ${ }^{2}$ \\ ${ }^{1}$ Eng $^{\mathrm{a}}$ Civil, Dra ${ }^{\mathrm{a}}$, Depto. de Engenharia Civil, UTFPR, Pato Branco, PR, Brasil - jabur@utfpr.edu.br \\ ${ }^{2}$ Eng. Florestal, Dr., Depto. de Engenharia e Tecnologia Florestal, UFPR, Curitiba, PR, Brasil - niva@ufpr.br \\ Recebido para publicação: 28/03/2010 - Aceito para publicação: 21/06/2011
}

\begin{abstract}
Resumo
O objetivo deste artigo é a estimativa da vazão máxima com o uso do método da curva número desenvolvido pelo Soil Conservation Service (SCS), pelo uso da fórmula racional e da equação de Ven Te Chow, ambos métodos hidrológicos que transformam chuva em vazão, tendo como estudo de caso a bacia hidrográfica do alto do rio Ligeiro (1/3 superior). A bacia hidrográfica localiza-se no município de Pato Branco (latitude 2609'52" Sul e longitude 52 $41^{\prime} 44^{\prime \prime}$ Oeste) e apresenta uma área de 68,26 km². Para obtenção do modelo hidrológico, foi necessária a confecção de mapas temáticos de uso do solo para os anos de 1980 e 2008. Nas condições de umidade normal $\left(\mathrm{N}_{\text {III }}\right)$, tempo de recorrência de 10 anos, a vazão de pico (chuva 0,3h) elevou-se de $59,76 \mathrm{~m}^{3}$ em 1980 para 72,13 $\mathrm{m}^{3}$ em 2008 . Essa vazão é consequência da impermeabilização da bacia no período e redução da área de cultivos agrícolas que, consequentemente, reduziram as taxas médias de infiltração da bacia (as áreas cultivadas em 1980 atingiam 70,63\% do total da bacia e em 2008 foram reduzidas a um percentual de 51,98\%).

Palavras-chave: Hidrologia; bacia hidrográfica; rio Ligeiro; vazão e curva número.
\end{abstract}

\begin{abstract}
Estimates of maximum rate and water saturation capacity of Upper River Ligeiro watershed, in Pato Branco, $P R$. The aim of this paper is to estimate the maximum flow in Upper River Ligeiro watershed (1/3 higher) using the curve number method, developed by Soil Conservation Service (SCS), by the application of the rational formula and the Ven Te Chow equation, both hydrologic methods that transform rainfall into runoff. This watershed is located in Pato Branco (latitude 26 09'52" S and longitude $52^{\circ} 41^{\prime} 44^{\prime \prime} \mathrm{W}$ ) covering an area of $68.26 \mathrm{~km}^{2}$. In order to get the hydrological model it was necessary the preparation of thematic maps of land use for the years 1980 and 2008. Under normal humidity conditions $\left(\mathrm{N}_{\mathrm{II}}\right)$, recurrence time of 10 years, the peak flow (rainfall $0.3 \mathrm{~m}$ ) increased from $59.76 \mathrm{~m}^{3}$ in 1980 to $72.13 \mathrm{~m}^{3}$ in 2008 . This flow is due to the impermeability of the basin in the period and agricultural crops area reducing, which consequently reduced the average rate of infiltration basin (the cultivated areas in 1980 reached $70.63 \%$ of the total watershed and in 2008 were reduced to a percentage of $51.98 \%$ ).

Keywords: Hydrology; watershed; river Ligeiro; flow and curve number.
\end{abstract}

\section{INTRODUÇÃO}

A modificação das características naturais de uma bacia hidrográfica impacta no funcionamento hidrológico da mesma, com a expansão do perímetro urbano induzindo, pela impermeabilização da superfície, altos índices de enchentes urbanas, como consequência do excesso de escoamento superficial produzido pela redução da capacidade de infiltração no solo.

A urbanização altera o ciclo hidrológico das bacias hidrográficas através de várias modificações. $\mathrm{O}$ aumento da impermeabilização faz com que o escoamento superficial obtenha altas velocidades e aumento de volume. Picos de vazão ocorrem com mais frequência e em grandes magnitudes, em comparação com áreas florestais ou rurais. Em adição, as enchentes ocorrem mais frequentemente, sendo que a bacia torna-se mais sensível às precipitações tanto moderadas como fracas (WATTS; HAEKE, 2003). 
Os estudos hidrológicos exigem, em sua maioria, a manipulação de dados de vazão e sua representação gráfica, para que se tenha uma visão do comportamento hídrico do rio para o uso em obras hidráulicas (PINHEIRO; BADIA, 2008).

O presente estudo teve por objetivo principal a estimativa da vazão máxima com a aplicação do método da curva número, também denominado números hidrológicos, desenvolvido pelo SCS, atualmente Natural Resource Conservation Service (NRCS), com aplicação da fórmula racional e da equação de Ven Te Chow (CHOW et al., 1994).

\section{MATERIAIS E MÉTODO}

\section{Caracterização da área de estudo}

A área de estudo - 1/3 superior da bacia hidrográfica do alto do rio Ligeiro (Figura 1) incorpora em seu espaço físico a urbanização da cidade de Pato Branco, no sudoeste do Paraná. O rio Ligeiro é afluente da margem esquerda da bacia hidrográfica do rio Chopim e este, por sua vez, afluente da margem esquerda da bacia hidrográfica do rio Iguaçu. A bacia do rio Iguaçu é a maior do estado do Paraná, com área de $70.800 \mathrm{~km}^{2}$ e $80,4 \%$ correspondendo a 16,6\% do estado do Paraná (SECRETARIA DE MEIO AMBIENTE E RECURSOS HÍDRICOS - SEMA, 2000). A cidade de Pato Branco se localiza a $432 \mathrm{~km}$ de Curitiba, entre as cidades de Francisco Beltrão e Coronel Vivida, que integram a região do sudoeste do estado do Paraná (TABALIPA, 2008).

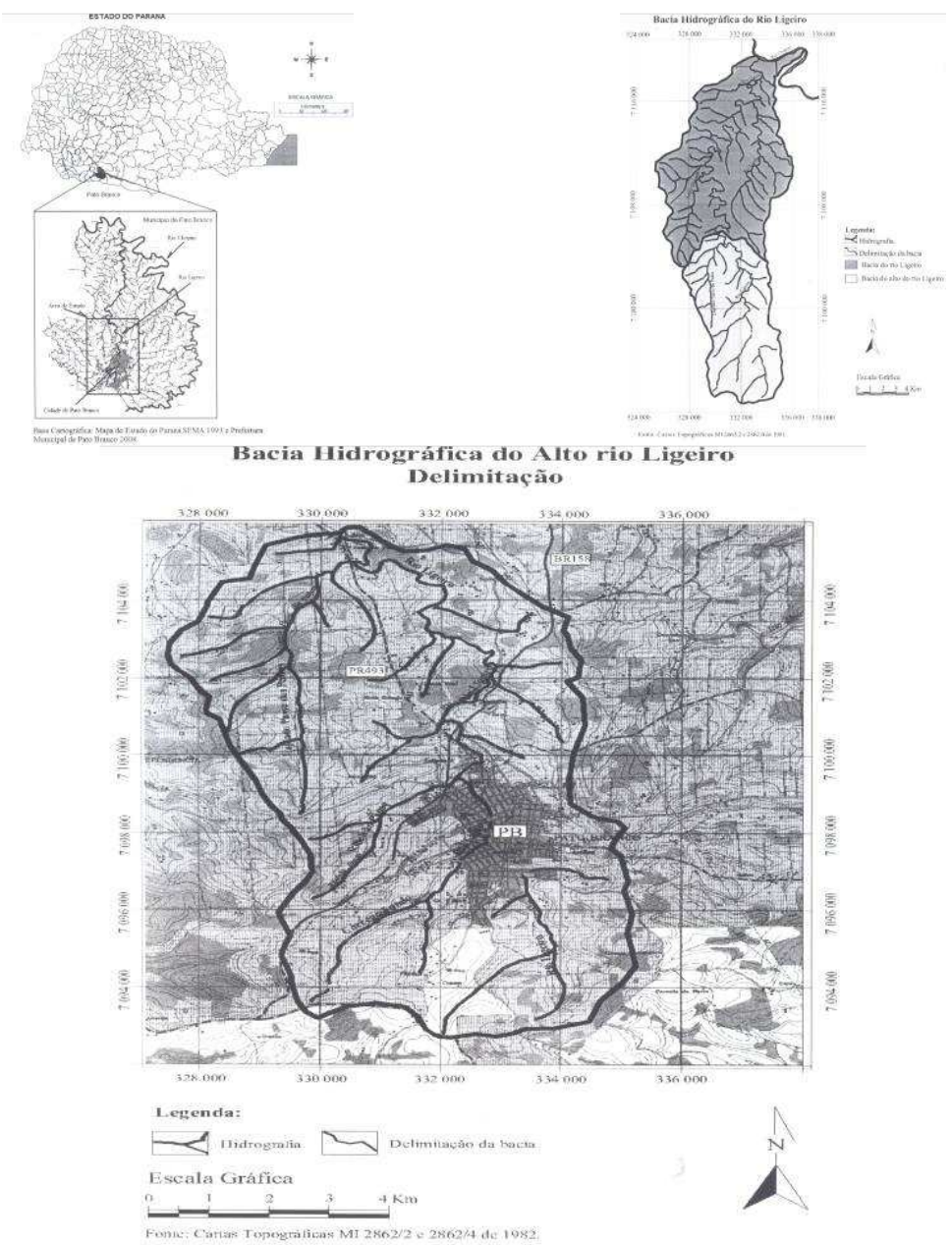

Figura 1. Localização e detalhe da área de estudo.

Figure 1. Location and detail study area. 
Para a confecção dos mapas, utilizaram-se as cartas topográficas MI - 2862/2 e MI - 2862/4, ambas de 1982, na escala 1:50.000. Utilizaram-se mapas digitalizados e fotografias áreas cedidas pelo Instituto de Terras, Cartografia e Geociências do Paraná (ITCG) e mapas de ocupação urbana fornecidos pelo Instituto de Pesquisa e Planejamento Urbano de Pato Branco (IPPUPB). A partir dessa base cartográfica, delimitou-se a bacia hidrográfica e se confeccionou o mapa de uso do solo para os anos de 1980 e 2008, os quais serviram para aplicação do modelo hidrológico.

\section{Determinação da chuva-vazão}

O método racional é considerado o melhor e mais simples modelo para estimativas de picos de vazão e dimensionamento de obras de drenagem. O pico de vazão do exutório da bacia hidrográfica é calculado em função da área, do coeficiente de escoamento (runoff) e da intensidade da precipitação (WATTS; HAWKE, 2003), sendo indicado para bacias hidrográficas de até $4 \mathrm{~km}^{2}$ (TUCCI, 1997):

$$
Q=0,278 \cdot C \cdot i \cdot A \quad \text { Equação (1) }
$$

em que: $\mathrm{Q}=$ vazão $\left(\mathrm{m}^{3} / \mathrm{s}\right)$;

$\mathrm{C}=$ coeficiente de escoamento superficial (runoff);

$\mathrm{i}$ = intensidade da precipitação conforme o tempo de concentração $(\mathrm{mm} / \mathrm{h})$;

$\mathrm{A}=$ área da bacia $\left(\mathrm{km}^{2}\right)$.

A fórmula da intensidade de precipitação máxima (i) é calculada para cada cidade, conforme a sua fórmula-base regionalizada (SUPERINTENDÊNCIA DE DESENVOLVIMENTO DE RECURSOS HÍDRICOS E SANEAMENTO AMBIENTAL - SUDERHSA, 2009), segundo a equação:

$$
i=\frac{a}{(t+b)^{m}} \cdot \operatorname{Tr}^{n}
$$

em que: $\mathrm{i}=$ intensidade pluviométrica $(\mathrm{mm} / \mathrm{h})$;

$\mathrm{td}=$ duração da precipitação (min.);

$\operatorname{Tr}=$ tempo de recorrência (anos);

$\mathrm{a}, \mathrm{b}, \mathrm{m}, \mathrm{n}=$ coeficientes definidos pela fórmula-base regionalizada.

Outro método também muito utilizado é o proposto por Ven Te Chow, o qual apresenta estimativas de vazões máximas, ou seja, das vazões de projeto para a previsão de enchentes e elaboração de obras hidráulicas. Essa estimativa é feita com base de dados de chuvas intensas que ocorrem na respectiva bacia em estudo. Esse método utiliza as hipóteses de hidrograma unitário, considerando que o fenômeno de transformação da chuva em vazão é regido por equações lineares. Nesse método, as vazões máximas são proporcionais às chuvas efetivas (NUNES; FIORI, 2007). A equação que foi desenvolvida para a vazão de pico, pelo método hidrológico de Ven Te Chow, que depende da área da bacia hidrográfica, é:

$$
Q=\frac{A \cdot X \cdot Y \cdot Z}{3,6}
$$

em que: $\mathrm{Q}=$ vazão máxima $\mathrm{em} \mathrm{m}^{3} / \mathrm{s}$;

Equação (3)

$\mathrm{A}=$ área da bacia em $\mathrm{km}^{2}$;

$\mathrm{X}$ = fator de deflúvio, sendo igual à razão da precipitação efetiva pela duração da chuva (Pe/td);

$\mathrm{Y}=$ fator climático (igual à unidade em locais onde há fórmula-base regionalizada);

$\mathrm{Z}=$ fator de redução do tempo de pico (adimensional).

O valor do fator de atenuação ou redução $(Z)$ de pico é definido como a relação entre tempo de pico (tp), o qual é calculado conforme a equação 4, e duração da chuva (td) (NUNES; FIORI, 2007):

$$
t p=0,005055 \cdot\left(\frac{L}{\sqrt{I}}\right)^{0,64}
$$

em que: tp = tempo de pico da vazão ou de pico (horas);

$\mathrm{L}=$ comprimento do curso d'água principal $(\mathrm{km})$;

$\mathrm{I}=$ declividade média do curso d'água principal (\%). 
Segundo Mendes Filho et al. (2007), os solos são classificados de acordo com a suscetibilidade à erosão e à produção de escoamento, de acordo com suas propriedades hidrológicas, independentemente da cobertura e da declividade da bacia. Na classificação original, o SCS reuniu os solos dos Estados Unidos em quatro grupos, baseando-se na premissa de que os perfis de solo com características semelhantes (espessura, textura, conteúdo de matéria orgânica, estrutura e grau de expansão, sendo a cada um deles atribuído uma letra A, B, C ou D) (Tabela 1) respondem de forma semelhante a uma chuva de grande duração e intensidade considerável, ou seja, conforme a capacidade de infiltração e produção de escoamento.

Tabela 1. Classificação dos solos segundo a capacidade de infiltração (classe hidrológica).

Table 1. Soil classification according to infiltration capacity (hydrological class).

\begin{tabular}{lcc} 
Características físicas e estruturais dos solos & $\begin{array}{c}\text { Capacidade } \\
\text { de infiltração } \\
(\mathbf{m m . h})\end{array}$ & $\begin{array}{c}\text { Classes } \\
\text { hidrológicas } \\
\text { dos solos }\end{array}$ \\
\hline Solos arenosos, profundos e bem drenados & $>7,6$ & $\mathrm{~A}$ \\
Solos arenosos com pouca argila e solo orgânico & 3,8 e 7,6 & $\mathrm{~B}$ \\
Solos mais argilosos que aqueles do grupo B, com baixa permeabilidade & 1,3 e 3,8 & $\mathrm{C}$ \\
Solos com argilas pesadas, muito impermeáveis & $<1,3$ & $\mathrm{D}$ \\
\hline
\end{tabular}

Fonte: Mendes et al. (2007).

O método da curva número foi apresentado em 1972 pelo SCS (Secretaria de Agricultura dos Estados Unidos) (LANÇA; RODRIGUES, 2000). A metodologia da curva número (CN) do SCS se baseia na classificação hidrológica do solo (conforme seu grupo hidrológico), sua utilização (uso do solo) e a condição de sua superfície no que diz respeito à potencialidade de gerar escoamento superficial (BAUNGARTEN et al. 2003).

Watts e Hawke (2003) consideram que a CN é melhor de ser trabalhada do que o coeficiente de escoamento (runoff) utilizado no método racional para estimativa de picos de vazão, pois este coeficiente apresenta alta incerteza, diferenciando-se da $\mathrm{CN}$, que mostra uma forte correlação entre o uso do solo e a resposta hidrológica. A expressão proposta para o cálculo da precipitação efetiva - que produz escoamento direto - pelo método da CN (LANÇA; RODRIGUES, 2000) é a seguinte:

$$
P e=\frac{\left(P p-\frac{5080}{C N}+50,8\right)^{2}}{P p+\frac{20320}{C N}+203,2}
$$

em que: $\mathrm{P}_{\mathrm{e}}=$ precipitação efetiva; que produz escoamento direto $(\mathrm{Qd})$;

$\mathrm{Pp}=$ precipitação total;

$\mathrm{CN}$ = curva número.

Os parâmetros $\mathrm{CN}$ adquiriram valores compreendidos entre 0 a 100 e dependem do tipo de solo e do teor de umidade (BAUNGARTEN et al., 2003; LANÇA; RODRIGUES, 2000). Solos com valores de $\mathrm{CN}$ tendendo a 100 são considerados impermeáveis, e CN tendendo a zero são completamente permeáveis. Segundo Baungarten et al. (2003), a partir de estudos desenvolvidos pelo SCS em diferentes bacias, tanto urbanas como rurais, foi possível tabelar valores de $\mathrm{CN}$ para diversos tipos de solo, segundo a sua capacidade de infiltração e interação com as tipologias de cobertura do solo.

Os valores da $\mathrm{CN}$ são valores relativos às condições antecedentes de umidade do solo referentes à situação, denominada de $\mathrm{N}_{\text {II }}$, ou seja, em condições normais de umidade do solo. Essas condições antecedentes de umidade são ajustadas através de relações com as condições de normalidade de umidade, segundo a tabela 2 (BAUNGARTEN et al., 2003). Para cálculo de novos valores, para condições $\mathrm{N}_{\mathrm{I}} \mathrm{e}$ $\mathrm{N}_{\text {III }}$, são utilizadas as equações (6) e (7) a seguir. A tabela 3 apresenta valores de $\mathrm{CN}$ para áreas urbanas e suburbanas e exclusivamente para áreas rurais com atividades agrícolas, de pastagens e de floresta, em condições de umidade antecedente média $\left(\mathrm{N}_{\mathrm{II}}\right)$. 
Tabela 2. Condições antecedentes de umidade.

Table 2. Humidity conditions background.

$\mathbf{N}_{\mathbf{I}} \quad$ Situação em que os solos estão secos, mas ainda não no ponto de murcha. A consideração desse caso é pouco recomendável para estudos de vazões de cheias.

$\mathbf{N}_{\text {II }} \quad$ Situação média em que a umidade do solo deve corresponder à capacidade de campo. Essa situação corresponde provavelmente às condições de umidade antecedentes de cheias de pequenas dimensões.

$\mathbf{N}_{\text {III }} \quad$ Situação em que ocorreram precipitações consideráveis nos cincos dias anteriores e o solo se encontra quase saturado. Essa situação é mais propícia à formação de maiores cheias e, portanto aquela que tem maior importância para o projeto.

Fonte: Baungarten et al. (2003).

$$
\begin{array}{lll}
\text { Condição I: } & N_{I}=\frac{4,2 \cdot N_{I I}}{10-0,058 \cdot N_{I I}} & \text { Equação (6) } \\
\text { Condição III: } & N_{I I I}=\frac{23 \cdot N_{I I}}{10+0,13 \cdot N_{I I}} & \text { Equação (7) }
\end{array}
$$

em que: $\mathrm{N}_{\mathrm{II}}=$ número da $\mathrm{CN}$ na tabela da condição de umidade normal.

\begin{tabular}{|c|c|c|c|c|}
\hline \multirow{2}{*}{ Cobertura do solo } & \multicolumn{4}{|c|}{ Classes hidrológicas dos solos } \\
\hline & $\mathbf{A}$ & B & $\mathbf{C}$ & D \\
\hline \multicolumn{5}{|l|}{ Áreas de agricultura } \\
\hline Sem medidas de conservação do solo & 72 & 81 & 88 & 91 \\
\hline Com medidas de conservação do solo & 62 & 71 & 78 & 81 \\
\hline \multicolumn{5}{|l|}{ Pastagem ou terrenos baldios } \\
\hline Em más condições & 68 & 79 & 86 & 89 \\
\hline Em boas condições & 39 & 61 & 74 & 80 \\
\hline Prados em boas condições & 30 & 58 & 71 & 78 \\
\hline \multicolumn{5}{|l|}{ Bosques ou áreas florestais } \\
\hline Cobertura sem matéria orgânica ("Mulch”) & 45 & 66 & 77 & 83 \\
\hline Boa cobertura do solo & 25 & 55 & 70 & 77 \\
\hline \multicolumn{5}{|c|}{ Espaços abertos, relvados, parques, campo de golfe, cemitério etc. } \\
\hline Boas condições, com relva cobrindo mais de $75 \%$ da área & 39 & 61 & 74 & 80 \\
\hline Condições razoáveis, com relva cobrindo de 50 a $75 \%$ da área & 49 & 69 & 79 & 84 \\
\hline \multicolumn{5}{|l|}{ Áreas comerciais e industriais } \\
\hline Áreas comerciais e de escritórios (85\% da área impermeável) & 89 & 92 & 94 & 95 \\
\hline Áreas industriais (72\% de área impermeável) & 81 & 88 & 91 & 93 \\
\hline \multicolumn{5}{|l|}{ Áreas residenciais } \\
\hline Áreas médias dos lotes $<500 \mathrm{~m}^{2}$ com média de $65 \%$ impermeável & 77 & 85 & 90 & 92 \\
\hline Áreas médias dos lotes até $1000 \mathrm{~m}^{2}$ com média de $38 \%$ impermeável & 61 & 75 & 83 & 87 \\
\hline Áreas médias dos lotes até $1300 \mathrm{~m}^{2}$ com média de $30 \%$ impermeável & 57 & 72 & 81 & 86 \\
\hline Áreas médias dos lotes até $2000 \mathrm{~m}^{2}$ com média de $25 \%$ impermeável & 54 & 70 & 80 & 85 \\
\hline Áreas médias dos lotes até $4000 \mathrm{~m}^{2}$ com média de $20 \%$ impermeável & 51 & 68 & 79 & 84 \\
\hline \multicolumn{5}{|l|}{ Superficies variadas } \\
\hline Parques de estacionamentos, telhados, viadutos etc. & 98 & 98 & 98 & 98 \\
\hline \multicolumn{5}{|l|}{ Arruamentos e estradas } \\
\hline Asfaltadas e com drenagem de águas pluviais & 98 & 98 & 98 & 98 \\
\hline Gravilha ou macadamizadas & 76 & 85 & 89 & 91 \\
\hline Terra & 72 & 82 & 87 & 89 \\
\hline
\end{tabular}

Tabela 3. Valores de curva número para diferentes recobrimentos em áreas urbanas e suburbanas.

Table 3. Numbers curves values from different urban and suburban areas.

Fonte: Baungarten et al. (2003). expressão:

Para determinação da capacidade máxima de retenção de água de uma bacia, utiliza-se a seguinte 


$$
S=\frac{25400}{C N}-254
$$

em que: $\mathrm{S}=$ capacidade de retenção máxima de saturação da bacia (mm);

Equação (8)

$\mathrm{CN}=$ valor da curva número.

O escoamento superficial não se inicia logo após a precipitação, isso vai depender da saturação na qual se encontra o solo, da interceptação e da retenção temporária de água na bacia. A altura da água precipitada até o início da formação do escoamento superficial constitui o que a SCS denomina de perdas iniciais, representada por 'It'. Essas perdas iniciais são constituídas por três parcelas: interceptação, retenção e infiltração até a saturação da camada superficial do solo (BAUNGARTEN et al., 2003).

\section{RESULTADOS E DISCUSSÃO}

\section{Intensidade de precipitação máxima}

A intensidade da chuva para os tempos de duração e tempo de recorrências analisados estão apresentados na tabela 4. A equação de intensidade pluviométrica foi obtida na carta de intensidade pluviométrica da SUDERHSA (2000). Recorrendo à equação-base regionalizada, obtém-se para Pato Branco, em latitude $26^{\circ} 07^{\prime} 00^{\prime \prime}$ e longitude 52 $41^{\prime} 00^{\prime \prime}: \mathrm{a}=879,43 ; \mathrm{m}=0,152 ; \mathrm{b}=9 ; \mathrm{n}=0,732$.

$$
i=\frac{879,43}{(t d+9)^{0,732}} \cdot \operatorname{Tr}^{0,152} \quad \text { Equação (9) }
$$

Com a equação da intensidade da chuva, adotou-se o tempo de recorrência para os seguintes valores: 10, 25, 50 e 100 anos, respectivamente, pois esses anos são, na sua maioria, utilizados para obras hidráulicas. O tempo da duração da precipitação também foi adotado com os seguintes valores: 20,40 , $60,80,100,120,140,160,210$ e 240 minutos. Os valores obtidos estão na tabela 4.

Tabela 4. Precipitação "i” (mm.h $\left.{ }^{-1}\right)$, para tempos de recorrência "Tr"e duração de precipitação "td" (min.).

\begin{tabular}{|c|c|c|c|c|}
\hline $\begin{array}{l}\text { Tempo (min.) } \\
\text { "td" }\end{array}$ & $\begin{array}{c}\text { Tempo de } \\
\text { recorrência } \\
\operatorname{Tr}=10 \text { anos } \\
(\mathrm{mm} / \mathrm{h})\end{array}$ & $\begin{array}{c}\text { Tempo de } \\
\text { recorrência } \\
\operatorname{Tr}=25 \text { anos } \\
(\mathrm{mm} / \mathrm{h})\end{array}$ & $\begin{array}{c}\text { Tempo de } \\
\text { recorrência } \\
\operatorname{Tr}=\mathbf{5 0} \text { anos } \\
(\mathbf{m m} / \mathbf{h})\end{array}$ & $\begin{array}{c}\text { Tempo de } \\
\text { recorrência } \\
\operatorname{Tr}=100 \text { anos } \\
(\mathrm{mm} / \mathrm{h})\end{array}$ \\
\hline 20 & 106,10 & 121,96 & 135,51 & 150,57 \\
\hline 40 & 72,27 & 83,07 & 92,30 & 102,56 \\
\hline 60 & 56,26 & 64,66 & 71,85 & 79,83 \\
\hline 80 & 46,69 & 53,67 & 59,63 & 66,26 \\
\hline 100 & 40,25 & 46,27 & 51,41 & 57,12 \\
\hline 120 & 35,58 & 40,90 & 45,45 & 50,50 \\
\hline 160 & 32,02 & 36,81 & 40,90 & 45,44 \\
\hline 180 & 29,20 & 33,56 & 37,29 & 41,44 \\
\hline 210 & 24,15 & 27,76 & 30,85 & 34,28 \\
\hline 240 & 21,99 & 25,27 & 28,08 & 28,71 \\
\hline
\end{tabular}
Table 4. Rainfall intensity values $\left(\mathrm{mm} \cdot \mathrm{h}^{-1}\right)$, to from recurrence times and duration of precipitation.

Os resultados obtidos apresentam a estimativa de intensidade de chuva $(\mathrm{mm} / \mathrm{h})$ para cada tempo de recorrência. Quanto maior o tempo de recorrência adotado, maior é a intensidade da chuva a ser trabalhada em projeto, isso porque a equação da intensidade de chuva é diretamente proporcional ao tempo de recorrência. Para tempo de $\mathrm{Tr}=10$ anos e $\mathrm{td}=20$ minutos, a intensidade de chuva resultou em $106,10 \mathrm{~mm} / \mathrm{h}$, enquanto que, para a mesmo tempo de duração e $\operatorname{Tr}=100$ anos, a intensidade da chuva resultou em 150,57 mm/h, com acréscimo de $44,41 \mathrm{~mm} / \mathrm{h}$. Para projetos hidráulicos, o engenheiro deve estar consciente do tempo de retorno a ser adotado, para que não ocorram falhas, como transbordamentos de canais ou subdimensionamento de tubulações. 


\section{Enquadramento das Curvas Números}

Para a classificação hidrológica do solo, utilizou-se o trabalho elaborado por Tapalipa (2008), o qual estudou a estabilidade de vertentes na bacia hidrográfica do rio Ligeiro. Neste estudo, para definição das classes hidrológicas dos solos, foram amostrados 16 pontos, de diferentes profundidades, retiradas amostras de solo e levadas até o laboratório, onde se determinou a granulometria ou forma granulométrica por peneiramento e sedimentação (areia, silte e argila). De posse dos dados de cada amostra, processou-se o enquadramento nas classes hidrológicas de solo (A, B, C, D), conforme preconiza a metodologia. Cada resultado das amostras foi inserido no ábaco e obtido o grupo hidrológico do solo. Na bacia hidrográfica em estudo, dos quatros grupos descritos pelo método SCS, foi identificado apenas 1 grupo, o grupo D, constituído com elevado potencial de escoamento e baixa taxa de infiltração.

Diversas classes de uso do solo foram estipuladas para se identificarem as áreas de cada um e também para se determinar a CN para cada tipologia, em tabela para bacias urbanas e suburbanas. A tipologia da cobertura do solo do terreno e a alteração ao longo dos dois períodos estudados (Tabela 5) foram quantificadas através dos mapas dos anos de 1980 e 2008.

Como a bacia em estudo apresenta diferentes tipos de solo e de cobertura superficial, foi necessário adotar um único valor da $\mathrm{CN}$ para cada ano estudado, o qual foi obtido pela média ponderada dos diversos valores de $\mathrm{CNs}$ correspondentes às tipologias de uso do solo. Pode-se observar que, na situação $\mathrm{N}_{\mathrm{III}}$, o valor médio da $\mathrm{CN}$ foi o maior, devido à situação de saturação do solo, bem como à impermeabilização ocorrida.

\section{Capacidade de saturação da bacia (S)}

Os resultados das áreas entre os anos de 1980 e 2008 mostram uma pequena redução da vegetação local, decorrente do crescimento da cidade para a região sul, onde se localizam os bairros de baixa densidade, e no perímetro mais central, onde se localiza a área de comércio e de serviços.

A área central da cidade, no período de 1980 a 2008 , cresceu apenas $0,18 \mathrm{~km}^{2}$, o que mostra a saturação dos espaços urbanos centrais atualmente. Por outro lado, houve uma expansão da área industrial de produção de bens e produtos, da indústria agropecuária e de serviços de informática, crescimento este, explicado em grande parte pela transformação da área agrícola. A expansão de áreas de serviços está representada, em grande parte, também pelas áreas de ensino, universidades e faculdades, que se estabeleceram depois de 1980.

Tabela 5. Uso do solo da bacia do rio Ligeiro e valores médios de CN nos anos de 1980 e 2008.

Table 5. Use of the soil of the Ligeiro River watershed and average values CN in the years 1980 and 2008.

\begin{tabular}{|c|c|c|c|c|c|c|c|}
\hline \multirow{3}{*}{ Tipologias de uso do solo } & \multicolumn{4}{|c|}{ Áreas } & \multicolumn{3}{|c|}{$\begin{array}{c}\text { CN em situação } \\
\text { de } N_{\text {I }} \text { para classe } \\
\text { hidrológica } D\end{array}$} \\
\hline & \multicolumn{2}{|c|}{1980} & \multicolumn{2}{|c|}{2008} & \multirow[b]{2}{*}{$\mathbf{N}_{\mathbf{I}}$} & \multirow[b]{2}{*}{$\mathbf{N}_{\text {II }}$} & \multirow{2}{*}{$\mathbf{N}_{\text {II }}$} \\
\hline & $\mathbf{k m}^{2}$ & $\%$ & $\mathbf{k m}^{2}$ & $\%$ & & & \\
\hline $\begin{array}{l}\text { Área com vegetação densa } \\
\text { Tabela 3: Boa cobertura do solo }\end{array}$ & 8,7 & 12,75 & 7,54 & 11,05 & 58 & 77 & 89 \\
\hline $\begin{array}{l}\text { Área com vegetação esparsa } \\
\text { Tabela 3: Cobertura má sem matéria orgânica }\end{array}$ & 3,6 & 5,27 & 4,58 & 6,71 & 67 & 83 & 92 \\
\hline $\begin{array}{l}\text { Área residencial baixa (média de } 30 \% \text { impermeável) } \\
\text { Tabela 3: Áreas médias dos lotes de } 1000 \mathrm{~m}^{2}\end{array}$ & 3,05 & 4,47 & 8,06 & 11,81 & 74 & 87 & 94 \\
\hline $\begin{array}{l}\text { Área residencial média (média de } 65 \% \text { impermeável) } \\
\text { Tabela 3: Áreas médias dos lotes de }<500 \mathrm{~m}^{2}\end{array}$ & 3,4 & 4,98 & 6,24 & 9,14 & 83 & 92 & 96 \\
\hline $\begin{array}{l}\text { Área comercial (média 85\% impermeável) } \\
\text { Tabela 3: Áreas comerciais e residenciais }\end{array}$ & 1 & 1,46 & 1,18 & 1,73 & 89 & 95 & 98 \\
\hline $\begin{array}{l}\text { Área industrial e serviços (média 75\% impermeável) } \\
\text { Tabela 3: Áreas industriais }\end{array}$ & 0,3 & 0,44 & 5,18 & 7,59 & 85 & 93 & 97 \\
\hline $\begin{array}{l}\text { Área cultivada (com conservação do solo) } \\
\text { Tabela 3: Com medidas de conservação do solo }\end{array}$ & 48,21 & 70,63 & 35,48 & 51,98 & 64 & 81 & 91 \\
\hline Total de área da bacia & 68,26 & 100 & 68,26 & 100 & -- & -- & -- \\
\hline
\end{tabular}

Em áreas urbanizadas, o incremento no pico de cheia está intimamente relacionado com a existência de sistemas de drenagem pluvial urbana que aceleram o escoamento superficial, transferindo 
fluxos de inundação para locais onde ainda há espaço não urbanizado, principalmente em áreas de rebaixamento de cota de altitude. A redução da área cultivada em 18,65\% no período de 1980 a 2008 contribuiu significativamente para esse processo.

As tipologias de uso do solo da bacia hidrográfica, resultante da confecção dos mapas de uso do solo, foram relacionadas em semelhança com o preconizado pelo método de curva número e correspondente número de curva, para as condições $\mathrm{N}_{\mathrm{I}}, \mathrm{N}_{\mathrm{II}}$ e $\mathrm{N}_{\mathrm{III}}$ (Tabela 5).

Como a bacia em estudo apresentou diferentes tipos de solos e tipologias de uso, adotou-se valor médio de curva número para os anos de 1980 e 2008 (Tabela 6). Os valores da curva número evidenciam uma tendência à impermeabilização. Nas condições de seca $\left(\mathrm{N}_{\mathrm{I}}\right)$, no período, passa de 65,27 para 68,48, reduzindo a capacidade de saturação em 18,24 mm $(135,15-116,91)$. Esse comportamento hidrológico é também observado para as condições de $\mathrm{N}_{\mathrm{II}}$ e $\mathrm{N}_{\mathrm{III}}$. As figuras 2 e 3 mostram os mapas de capacidade máxima de saturação em relação às tipologias de uso do solo nas condições de $\mathrm{N}_{\mathrm{II}}$.

Tabela 6. Capacidade de máxima saturação da bacia (S) nos anos de 1980 e de 2008.

Table 6. Maximum capacity of saturation of watershed (S) in the years of 1980 and 2008.

\begin{tabular}{|c|c|c|c|c|c|c|}
\hline \multirow[t]{2}{*}{ Tipologia de uso do solo } & \multicolumn{3}{|c|}{$\begin{array}{c}\text { Curva número em diferentes } \\
\text { classes hidrológicas }\end{array}$} & \multicolumn{3}{|c|}{$\begin{array}{l}\text { Capacidade máxima de } \\
\text { saturação da bacia (mm) }\end{array}$} \\
\hline & $\mathbf{C N}_{\mathrm{I}}$ & $\mathbf{C N}_{\text {II }}$ & $\mathbf{C N}_{\text {III }}$ & SI & SII & SIII \\
\hline \multicolumn{7}{|c|}{1980} \\
\hline Área com vegetação densa & 58 & 77 & 89 & 183,93 & 75,87 & 31,39 \\
\hline Área com vegetação esparsa & 67 & 83 & 92 & 125,10 & 52,02 & 22,09 \\
\hline $\begin{array}{l}\text { Área residencial baixa (média de } 30 \% \\
\text { impermeável) }\end{array}$ & 74 & 87 & 94 & 89,24 & 37,95 & 16,21 \\
\hline $\begin{array}{l}\text { Área residencial média (média de } 65 \% \\
\text { impermeável) }\end{array}$ & 83 & 92 & 96 & 52,02 & 22,09 & 10,58 \\
\hline Área comercial, escritório e residencial & 89 & 95 & 98 & 31,39 & 13,37 & 5,18 \\
\hline Área industrial e de serviços & 85 & 93 & 97 & 44,82 & 19,12 & 7,86 \\
\hline Área cultivada (com conservação do solo) & 64 & 81 & 91 & 142,88 & 59,58 & 25,12 \\
\hline Média ponderada de $\mathrm{CN}$ & 65,27 & 81,68 & 91,32 & 135,15 & 56,96 & 24,14 \\
\hline \multicolumn{7}{|c|}{2008} \\
\hline Áreas com boas coberturas de florestas & 58 & 77 & 89 & 183,93 & 75,87 & 31,39 \\
\hline Zona de cobertura ruim (vegetação) & 67 & 83 & 92 & 125,10 & 52,02 & 22,09 \\
\hline $\begin{array}{l}\text { Zona residencial baixa (média de } 30 \% \\
\text { impermeável) }\end{array}$ & 74 & 87 & 94 & 89,24 & 37,95 & 16,21 \\
\hline $\begin{array}{l}\text { Zona residencial média (média de } 65 \% \\
\text { impermeável) }\end{array}$ & 83 & 92 & 96 & 52,02 & 22,09 & 10,58 \\
\hline Zonas comerciais, escritórios e residenciais & 89 & 95 & 98 & 31,39 & 13,37 & 5,18 \\
\hline Zonas industriais e de serviços & 85 & 93 & 97 & 44,82 & 19,12 & 7,86 \\
\hline Zonas cultivadas (com conservação do solo) & 64 & 81 & 91 & 142,88 & 59,58 & 25,12 \\
\hline Média ponderada de $\mathrm{CN}$ & 68,48 & 83,56 & 91,83 & 116,91 & 49,97 & 22,60 \\
\hline
\end{tabular}

\section{Precipitação efetiva (Pe) e vazão máxima e mínima média}

$\mathrm{Pe}$ (ou Es) refere-se ao escoamento superficial real que se quer estimar. Esse escoamento superficial atinge o leito do rio como Qd (vazão direta) do hidrograma de vazão. Se o total de Es escoar pela superfície do solo, poderá produzir erosão. Em síntese, é a quantidade de água que sobra depois de certa quantidade de água ser "abstraída" e "infiltrada" na bacia. A obtenção da precipitação efetiva permite calcular o coeficiente de escoamento superficial (CE) pela fórmula racional (Tabela 7).

Os resultados obtidos na tabela 7 apresentam altos valores de vazões máximas: de $970,45 \mathrm{~m}^{3} / \mathrm{s}$ para o ano de 1980, com uma chuva de duração de 20 minutos, para $1048,97 \mathrm{~m}^{3} / \mathrm{s}$ para o ano de 2008 , com o acréscimo de 78,52 $\mathrm{m}^{3} / \mathrm{s}$. Observa-se que o CE tendeu a aumentar com o passar dos anos, de 1,35\% em 1980 para 3,1\% em 2008 (condição $\mathrm{N}_{\mathrm{I}}$ ), mostrando a impermeabilização do solo ocorrida na bacia hidrográfica. 


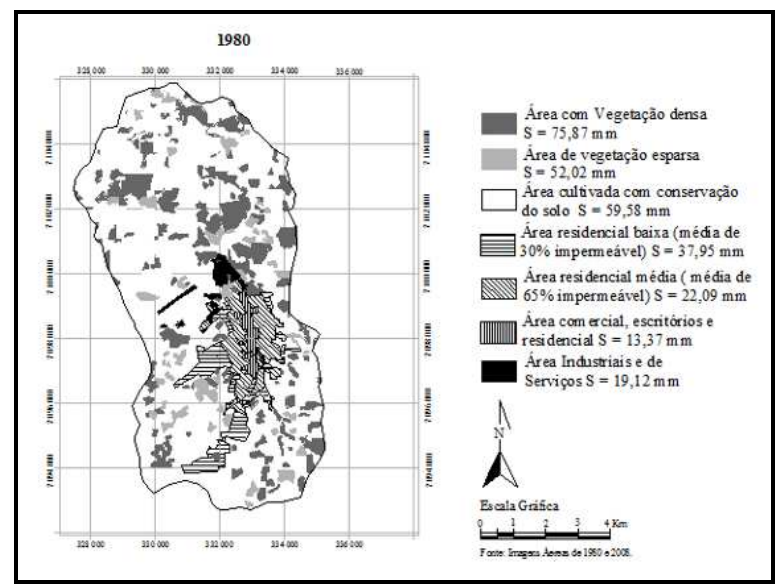

Figura 2. Mapa de capacidade máxima de saturação (S) da bacia no ano de 1980.

Figure 2. Map of maximum capacity of saturation (S) of watershed in the year 1980.

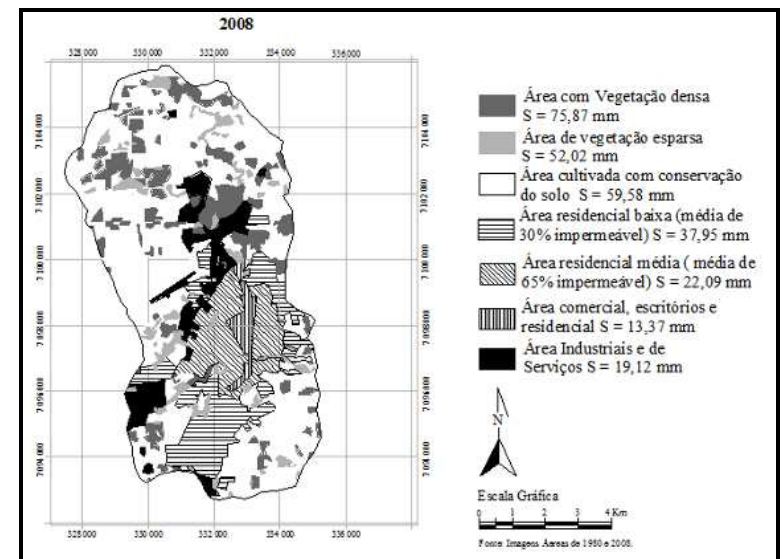

Figura 3. Mapa de capacidade máxima de saturação (S) da bacia no ano de 2008.

Figure 3. Map of maximum capacity of saturation (S) of watershed in the year 2008.

Tabela 7. Aplicação da fórmula racional tradicional de cálculo de vazão.

Table 7. Application of traditional rational formula of calculation flow.

\begin{tabular}{|c|c|c|c|c|c|c|c|c|}
\hline Ano & $\begin{array}{c}\text { Área } \\
\text { da } \\
\text { bacia } \\
\left(\mathbf{k m}^{2}\right)\end{array}$ & $\begin{array}{c}\mathrm{CN} \\
\text { média }\end{array}$ & $\begin{array}{c}\text { Classe } \\
\text { hidrológica }\end{array}$ & $\begin{array}{c}\text { Intensidade } \\
\text { chuva } \\
\text { Tr = 10 anos } \\
\text { ('6i') } \\
(\mathrm{mm} / \mathrm{h}) \\
\end{array}$ & $\begin{array}{c}\text { Capacidade } \\
\text { máxima de } \\
\text { saturação }\end{array}$ & $\begin{array}{c}\text { Precipitação } \\
\text { efetiva } \\
\text { Pe }(\mathbf{m m})\end{array}$ & $\begin{array}{c}\text { Coeficiente } \\
\text { de } \\
\text { escoamento } \\
\mathrm{CE}=\mathrm{Pe} / \mathrm{Pp}\end{array}$ & $\begin{array}{c}\text { Vazão } \\
\text { máxima } \\
\mathbf{Q} \\
\left(\mathbf{m}^{3} / \mathbf{s}\right)\end{array}$ \\
\hline \multirow{3}{*}{1980} & \multirow{6}{*}{68,26} & 65,27 & $\mathrm{~N}_{\mathrm{I}}$ & & 135,15 & 0,48 & 0,135 & 27,18 \\
\hline & & 81,68 & $\mathrm{~N}_{\mathrm{II}}$ & & 56,96 & 7,08 & 0,200 & 402,68 \\
\hline & & 91,32 & $\mathrm{~N}_{\text {II }}$ & 10610 & 24,14 & 17,06 & 0,482 & 970,45 \\
\hline \multirow{3}{*}{2008} & & 68,48 & $\mathrm{~N}_{\mathrm{I}}$ & 100,10 & 116,91 & 1,11 & 0,031 & 62,41 \\
\hline & & 83,56 & $\mathrm{~N}_{\text {II }}$ & & 49,97 & 8,54 & 0,242 & 487,24 \\
\hline & & 91,56 & $\mathrm{~N}_{\mathrm{II}}$ & & 22,60 & 18,41 & 0,521 & 1048,97 \\
\hline \multirow{3}{*}{1980} & \multirow{6}{*}{68,26} & 65,27 & $\mathrm{~N}_{\mathrm{I}}$ & & 135,15 & 18,93 & 0,215 & 89,72 \\
\hline & & 81,68 & $\mathrm{~N}_{\text {II }}$ & & 56,96 & 43,81 & 0,498 & 207,81 \\
\hline & & 91,32 & $\mathrm{~N}_{\text {II }}$ & & 24,14 & 64,41 & 0,732 & 305,46 \\
\hline \multirow{3}{*}{2008} & & 68,48 & $\mathrm{~N}_{\mathrm{I}}$ & 21,99 & 116,91 & 22,97 & 0,261 & 108,91 \\
\hline & & 83,56 & $\mathrm{~N}_{\mathrm{II}}$ & & 49,97 & 47,50 & 0,540 & 225,34 \\
\hline & & 91,56 & $\mathrm{~N}_{\mathrm{II}}$ & & 22,60 & 66,63 & 0,758 & 316,30 \\
\hline
\end{tabular}




\section{Tempo de máxima vazão (td) e vazão máxima (método Ven Te Chow)}

O método proposto por Ven Te Chow estabelece que a chuva efetiva ou chuva excedente que produz vazão é responsável pelas vazões de cheias, principalmente em bacias de pequenas escalas e urbanizadas, sendo justamente a impermeabilização do solo, em conjunto com a urbanização, a principal geradora do aumento do escoamento superficial. O comprimento do curso d'água da bacia é de 13,56 km e a declividade da bacia, verificada através da confecção do perfil longitudinal do fundo de vale do rio Ligeiro é de $1,18 \%$, logo, o "tp" da bacia hidrográfica é de 2 horas. Com os valores de "td" adotados desde o início do cálculo e com o tempo de pico determinado, obtém-se Z, segundo Winklen, 1971 (NUNES; FIORI, 2007) (Tabela 8).

Tabela 8. Fator de redução do tempo de pico de vazão.

Table 8. Time reduction factor of peak flow.

\begin{tabular}{lccccccccc}
\hline $\mathbf{t d} / \mathbf{t p}$ & $\mathbf{Z}$ & $\mathbf{t d} / \mathbf{t p}$ & $\mathbf{Z}$ & $\mathbf{t d} / \mathbf{t p}$ & $\mathbf{Z}$ & $\mathbf{t d} / \mathbf{t p}$ & $\mathbf{Z}$ & $\mathbf{t d} / \mathbf{t p}$ & $\mathbf{Z}$ \\
\hline 0,05 & 0,04 & 0,46 & 0,36 & 0,84 & 0,58 & 1,22 & 0,75 & 1,60 & 0,90 \\
0,10 & 0,08 & 0,48 & 0,38 & 0,86 & 0,59 & 1,24 & 0,76 & 1,62 & 0,90 \\
0,12 & 0,10 & 0,50 & 0,39 & 0,88 & 0,60 & 1,26 & 0,77 & 1,64 & 0,91 \\
0,14 & 0,12 & 0,52 & 0,40 & 0,90 & 0,60 & 1,28 & 0,78 & 1,66 & 0,91 \\
0,16 & 0,14 & 0,54 & 0,41 & 0,92 & 0,61 & 1,30 & 0,79 & 1,68 & 0,92 \\
0,18 & 0,16 & 0,56 & 0,42 & 0,94 & 0,62 & 1,32 & 0,80 & 1,70 & 0,92 \\
0,20 & 0,18 & 0,58 & 0,44 & 0,96 & 0,63 & 1,34 & 0,81 & 1,72 & 0,93 \\
0,22 & 0,19 & 0,60 & 0,45 & 0,98 & 0,64 & 1,36 & 0,82 & 1,74 & 0,93 \\
0,24 & 0,20 & 0,62 & 0,46 & 1,00 & 0,65 & 1,38 & 0,82 & 1,76 & 0,94 \\
0,26 & 0,22 & 0,64 & 0,47 & 1,02 & 0,66 & 1,40 & 0,82 & 1,78 & 0,94 \\
0,28 & 0,24 & 0,66 & 0,48 & 1,04 & 0,67 & 1,42 & 0,83 & 1,80 & 0,95 \\
0,30 & 0,25 & 0,68 & 0,49 & 1,06 & 0,68 & 1,44 & 0,84 & 1,82 & 0,95 \\
0,32 & 0,26 & 0,70 & 0,50 & 1,08 & 0,69 & 1,46 & 0,85 & 1,84 & 0,96 \\
0,34 & 0,28 & 0,72 & 0,51 & 1,10 & 0,70 & 1.48 & 0,86 & 1,86 & 0,96 \\
0,36 & 0,29 & 0,74 & 0,52 & 1,12 & 0,71 & 1,50 & 0,86 & 1,88 & 0,97 \\
0,38 & 0,30 & 0,76 & 0,54 & 1,14 & 0,72 & 1,52 & 0,87 & 1,90 & 0,97 \\
0,40 & 0,32 & 0,78 & 0,55 & 1,16 & 0,73 & 1,54 & 0,88 & 1,92 & 0,98 \\
\hline
\end{tabular}

Tabela 9. Valores de $\mathrm{Z}$ em função de $\mathrm{Td} / \mathrm{Tp}$.

Table 9. $\mathrm{Z}$ values in function of $\mathrm{Td} / \mathrm{Tp}$.

\begin{tabular}{lcc}
\hline $\begin{array}{l}\text { Tempo de duração td } \\
\text { em horas }\end{array}$ & td/tp & $\mathbf{Z}$ (fator de redução) \\
\hline 0,33 & 0,16 & 0,14 \\
0,67 & 0,32 & 0,26 \\
1,0 & 0,47 & 0,37 \\
1,33 & 0,63 & 0,465 \\
1,67 & 0,79 & 0,555 \\
2,0 & 0,95 & 0,625 \\
2,33 & 1,11 & 0,705 \\
2,67 & 1,27 & 0,775 \\
3,5 & 1,66 & 0,91 \\
4,0 & 1,89 & 0,97 \\
\hline
\end{tabular}

Exemplo de cálculo da tabela 9: para td de 0,3 horas, o valor da relação td/tp $=0,3 / 2,11=0,16$. Esse resultado compreende a $Z=0,14$. Os valores encontrados para $Z$ estão todos apresentados nessa tabela. Nota-se que, em alguns casos, para determinar $Z$ foi preciso interpolar os dados, como para $t d / t p=0,63$.

Após a obtenção dos valores de A, X, Y e Z, o último procedimento é calcular a vazão de pico para cada ano estabelecido. O procedimento foi repetido para todos os valores de duração da chuva (de 20 a 240 minutos) e em diferentes tempos de recorrência estipulados para estudos em obras de engenharia hidráulica e hidrológica: 10, 25, 50 e 100 anos. Os resultados finais, as vazões máximas simuladas para a bacia hidrográfica do rio Ligeiro dos anos de 1980 e 2008 e nos tempos de recorrências estipulados, estão apresentados nas tabelas 10 e 11 . 
Tabela 10. Precipitação efetiva $(\mathrm{mm})$ e vazão máxima $\left(\mathrm{m}^{3} / \mathrm{s}\right)$ pelo método de Ven Te Chow -1980 . Table 10. Efetive precipitation $(\mathrm{mm})$ and maxim flow $\left(\mathrm{m}^{3} / \mathrm{s}\right)$ the method from Ven Te Chow -1980.

\begin{tabular}{|c|c|c|c|c|c|c|}
\hline \multirow{2}{*}{$\begin{array}{l}\text { Tempo de } \\
\text { duração (h) } \\
\text { para } \\
\text { diferentes } \\
\text { tempos de } \\
\text { retorno } \\
\end{array}$} & \multicolumn{3}{|c|}{ Precipitação efetiva $(\mathrm{mm})$} & \multicolumn{3}{|c|}{ Vazão máxima Q $\left(\mathrm{m}^{3} / \mathrm{s}\right)$} \\
\hline & $\begin{array}{c}\text { Classe } \\
\text { hidrológica } \\
\mathbf{N}_{\mathrm{I}}(\mathbf{m m})\end{array}$ & $\begin{array}{c}\text { Classe } \\
\text { hidrológica } \\
\text { N }_{\text {II }}(\mathbf{m m})\end{array}$ & $\begin{array}{c}\text { Classe } \\
\text { hidrológica } \\
\text { N }_{\text {III }}(\mathbf{m m})\end{array}$ & $\begin{array}{c}\text { Classe } \\
\text { hidrológica } \\
\mathbf{N}_{\mathrm{I}}\left(\mathbf{m}^{3} / \mathbf{s}\right)\end{array}$ & $\begin{array}{c}\text { Classe } \\
\text { hidrológica } \\
\mathrm{N}_{\mathrm{II}}\left(\mathrm{m}^{3} / \mathrm{s}\right)\end{array}$ & $\begin{array}{c}\text { Classe } \\
\text { hidrológica } \\
\mathrm{N}_{\mathrm{III}}\left(\mathrm{m}^{3} / \mathrm{s}\right)\end{array}$ \\
\hline \multicolumn{7}{|c|}{ Tr 10 anos } \\
\hline 0,3 & 0,48 & 7,08 & 17,06 & 4,08 & 59,76 & 143,97 \\
\hline 0,7 & 2,86 & 14,40 & 27,85 & 22,31 & 112,85 & 218,28 \\
\hline 1,0 & 5,20 & 19,72 & 35,00 & 38,67 & 146,62 & 260,26 \\
\hline 1,3 & 7,28 & 23,94 & 40,43 & 51,12 & 167,79 & 283,40 \\
\hline 1,7 & 9,16 & 27,47 & 44,86 & 61,13 & 183,88 & 300,27 \\
\hline 2,0 & 10,87 & 30,54 & 48,64 & 68,34 & 191,82 & 305,49 \\
\hline 2,3 & 12,44 & 33,26 & 51,94 & 66,03 & 202,01 & 315,43 \\
\hline 2,7 & 13,90 & 35,73 & 54,89 & 72,12 & 208,68 & 320,65 \\
\hline 3,5 & 17,17 & 41,04 & 61,18 & 89,80 & 214,48 & 319,71 \\
\hline 4,0 & 18,93 & 43,81 & 64,41 & 92,22 & 213,55 & 313,95 \\
\hline \multicolumn{7}{|c|}{$\operatorname{Tr} 25$ anos } \\
\hline 0,3 & 1,25 & 9,93 & 21,40 & 10,53 & 83,81 & 180,66 \\
\hline 0,7 & 4,92 & 19,17 & 34,21 & 38,54 & 150,24 & 268,19 \\
\hline 1,0 & 8,20 & 25,74 & 42,63 & 60,95 & 191,42 & 317,03 \\
\hline 1,3 & 11,04 & 30,90 & 49,00 & 77,35 & 216,62 & 343,49 \\
\hline 1,7 & 13,54 & 35,20 & 54,19 & 90,64 & 235,62 & 362,68 \\
\hline 2,0 & 15,80 & 38,92 & 58,60 & 99,21 & 244,44 & 368,03 \\
\hline 2,3 & 17,85 & 42,21 & 62,45 & 108,41 & 256,30 & 379,24 \\
\hline 2,7 & 19,75 & 45,17 & 65,89 & 115,36 & 263,83 & 384,87 \\
\hline 3,5 & 23,97 & 51,55 & 73,21 & 125,24 & 269,37 & 382,55 \\
\hline 4,0 & 26,22 & 54,86 & 76,96 & 127,79 & 267,38 & 375,12 \\
\hline \multicolumn{7}{|c|}{$\operatorname{Tr} 50$ anos } \\
\hline 0,3 & 2,15 & 12,57 & 25,24 & 18,12 & 106,12 & 213,04 \\
\hline 0,7 & 7,02 & 23,47 & 39,77 & 55,01 & 183,99 & 311,77 \\
\hline 1,0 & 11,16 & 31,12 & 49,27 & 82,99 & 231,45 & 366,39 \\
\hline 1,3 & 14,68 & 37,09 & 56,44 & 102,89 & 260,01 & 395,60 \\
\hline 1,7 & 17,75 & 42,05 & 62,26 & 118,80 & 281,41 & 416,72 \\
\hline 2,0 & 20,49 & 46,31 & 67,21 & 128,71 & 290,87 & 422,13 \\
\hline 2,3 & 22,98 & 50,08 & 71,53 & 139,55 & 304,10 & 434,39 \\
\hline 2,7 & 25,27 & 53,46 & 75,39 & 147,58 & 312,30 & 440,34 \\
\hline 3,5 & 30,32 & 60,74 & 83,58 & 158,43 & 317,43 & 436,76 \\
\hline 4,0 & 33,00 & 64,52 & 87,78 & 160,86 & 314,46 & 427,86 \\
\hline \multicolumn{7}{|c|}{ Tr 100 anos } \\
\hline 0,3 & 3,39 & 15,72 & 29,60 & 28,60 & 125,16 & 249,90 \\
\hline 0,7 & 9,68 & 28,49 & 46,05 & 75,91 & 210,69 & 360,96 \\
\hline 1,0 & 14,83 & 37,35 & 56,74 & 110,30 & 262,01 & 421,93 \\
\hline 1,3 & 19,14 & 44,22 & 64,79 & 134,13 & 292,39 & 454,13 \\
\hline 1,7 & 22,86 & 49,89 & 71,32 & 152,98 & 315,03 & 477,35 \\
\hline 2,0 & 26,16 & 54,77 & 76,86 & 164,30 & 324,53 & 482,77 \\
\hline 2,3 & 29,14 & 59,07 & 81,70 & 176,95 & 338,41 & 496,16 \\
\hline 2,7 & 31,87 & 62,93 & 86,02 & 186,15 & 346,78 & 502,45 \\
\hline 3,5 & 37,87 & 71,21 & 95,18 & 197,89 & 351,05 & 497,39 \\
\hline 4,0 & 41,04 & 75,49 & 99,88 & 200,05 & 347,12 & 486,82 \\
\hline
\end{tabular}


Tabela 11. Precipitação efetiva $(\mathrm{mm})$ e vazão máxima $\left(\mathrm{m}^{3} / \mathrm{s}\right)$ pelo método de Ven Te Chow -2008 .

Table 11. Efetive precipitation $(\mathrm{mm})$ and maxim flow $\left(\mathrm{m}^{3} / \mathrm{s}\right)$ the method from Ven Te Chow -2008.

\begin{tabular}{|c|c|c|c|c|c|c|}
\hline \multirow{2}{*}{$\begin{array}{l}\text { Tempo de } \\
\text { duração (h) } \\
\text { para diferentes } \\
\text { tempos de } \\
\text { retorno }\end{array}$} & \multicolumn{3}{|c|}{ Precipitação efetiva (mm) } & \multicolumn{3}{|c|}{ Vazão máxima Q $\left(\mathrm{m}^{3} / \mathrm{s}\right)$} \\
\hline & $\begin{array}{c}\text { Classe } \\
\text { hidrológica } \\
\text { N }_{\mathrm{I}}(\mathrm{mm})\end{array}$ & $\begin{array}{c}\text { Classe } \\
\text { hidrológica } \\
\text { N }_{\text {II }}(\mathbf{m m})\end{array}$ & $\begin{array}{c}\text { Classe } \\
\text { hidrológica } \\
\mathrm{N}_{\text {III }}(\mathbf{m m})\end{array}$ & $\begin{array}{c}\text { Classe } \\
\text { hidrológica } \\
\mathrm{N}_{\mathrm{I}}\left(\mathbf{m}^{3} / \mathbf{s}\right)\end{array}$ & $\begin{array}{c}\text { Classe } \\
\text { hidrológica } \\
\text { N }_{\text {II }}\left(\mathbf{m}^{3} / \mathbf{s}\right)\end{array}$ & $\begin{array}{c}\text { Classe } \\
\text { hidrológica } \\
\mathrm{N}_{\text {III }}\left(\mathbf{m}^{\mathbf{3}} / \mathbf{s}\right)\end{array}$ \\
\hline \multicolumn{7}{|c|}{$\operatorname{Tr} 10$ anos } \\
\hline 0,3 & 1,11 & 8,54 & 18,41 & 9,41 & 72,13 & 155,44 \\
\hline 0,7 & 4,34 & 16,54 & 29,52 & 34,02 & 129,66 & 231,36 \\
\hline 1,0 & 7,21 & 22,24 & 36,82 & 53,65 & 165,37 & 273,80 \\
\hline 1,3 & 9,70 & 26,72 & 42,35 & 68,00 & 187,27 & 296,83 \\
\hline 1,7 & 11,89 & 30,45 & 46,85 & 79,60 & 203,77 & 313,55 \\
\hline 2,0 & 13,86 & 33,67 & 50,68 & 87,08 & 211,47 & 318,29 \\
\hline 2,3 & 15,66 & 36,52 & 54,02 & 95,11 & 221,78 & 328,06 \\
\hline 2,7 & 17,32 & 39,09 & 57,01 & 101,17 & 228,34 & 333,01 \\
\hline 3,5 & 21,00 & 44,63 & 63,37 & 109,76 & 233,21 & 331,13 \\
\hline 4,0 & 22,97 & 47,50 & 66,63 & 111,97 & 231,54 & 324,76 \\
\hline \multicolumn{7}{|c|}{$\operatorname{Tr} 25$ anos } \\
\hline 0,3 & 2,22 & 11,66 & 22,90 & 18,76 & 98,40 & 193,32 \\
\hline 0,7 & 6,88 & 21,60 & 36,02 & 53,90 & 169,33 & 282,34 \\
\hline 1,0 & 10,77 & 28,56 & 44,58 & 80,11 & 212,39 & 331,54 \\
\hline 1,3 & 14,06 & 33,98 & 51,05 & 98,55 & 238,20 & 357,80 \\
\hline 1,7 & 16,92 & 38,47 & 56,30 & 113,24 & 257,51 & 376,79 \\
\hline 2,0 & 19,47 & 42,34 & 60,75 & 122,26 & 265,94 & 381,58 \\
\hline 2,3 & 21,77 & 45,75 & 64,65 & 132,21 & 277,85 & 392,59 \\
\hline 2,7 & 23,89 & 48,82 & 68,12 & 139,53 & 285,19 & 397,91 \\
\hline 3,5 & 28,55 & 55,41 & 75,50 & 149,21 & 289,58 & 394,55 \\
\hline 4,0 & 31,03 & 58,83 & 79,29 & 151,24 & 286,73 & 386,46 \\
\hline \multicolumn{7}{|c|}{$\operatorname{Tr} 50$ anos } \\
\hline 0,3 & 3,42 & 14,53 & 26,84 & 28,89 & 122,66 & 226,59 \\
\hline 0,7 & 9,39 & 26,17 & 41,68 & 73,59 & 205,13 & 326,71 \\
\hline 1,0 & 14,20 & 34,21 & 51,32 & 105,62 & 254,41 & 381,61 \\
\hline 1,3 & 18,21 & 40,44 & 58,57 & 127,62 & 283,49 & 410,56 \\
\hline 1,7 & 21,66 & 45,59 & 64,46 & 144,96 & 305,12 & 431,42 \\
\hline 2,0 & 24,71 & 50,01 & 69,45 & 155,22 & 314,08 & 436,22 \\
\hline 2,3 & 27,47 & 53,90 & 73,81 & 166,79 & 327,31 & 448,24 \\
\hline 2,7 & 29,98 & 57,39 & 77,70 & 175,14 & 335,24 & 453,86 \\
\hline 3,5 & 35,51 & 64,88 & 85,95 & 185,56 & 339,06 & 449,17 \\
\hline 4,0 & 38,43 & 68,76 & 90,19 & 187,31 & 335,12 & 439,57 \\
\hline \multicolumn{7}{|c|}{$\operatorname{Tr} 100$ anos } \\
\hline 0,3 & 5,00 & 17,92 & 31,31 & 42,21 & 151,25 & 264,33 \\
\hline 0,7 & 12,50 & 31,45 & 48,05 & 98,00 & 246,55 & 376,65 \\
\hline 1,0 & 18,38 & 40,71 & 58,87 & 136,68 & 302,71 & 437,82 \\
\hline 1,3 & 23,20 & 47,84 & 67,01 & 162,65 & 335,33 & 469,70 \\
\hline 1,7 & 27,33 & 53,71 & 73,60 & 182,93 & 359,47 & 492,61 \\
\hline 2,0 & 30,96 & 58,74 & 79,19 & 194,48 & 368,92 & 497,38 \\
\hline 2,3 & 34,23 & 63,16 & 84,06 & 207,84 & 383,56 & 510,50 \\
\hline 2,7 & 37,20 & 67,13 & 88,41 & 217,28 & 392,10 & 516,41 \\
\hline 3,5 & 43,70 & 75,61 & 97,63 & 228,34 & 395,13 & 510,18 \\
\hline 4,0 & 47,12 & 80,00 & 102,36 & 229,64 & 389,89 & 498,88 \\
\hline
\end{tabular}

Os resultados apresentados nas tabelas 10 e 11 distinguem os anos de 1980 e 2008, mostrando que, com os passar de 28 anos, a precipitação efetiva ou escoamento superficial gerado sofreu um leve 
aumento, ocasionado pela modificação do uso do solo da bacia. Como exemplo, em td $=20$ minutos (0,33 horas) e $\operatorname{Tr}$ de 10 anos na condição $\mathrm{CN}_{\mathrm{I}}$, a Pe (precipitação efetiva ou escoamento superficial) resultou em 0,48 mm. Em comparação desses mesmos dados com 2008, a Pe subiu para 1,11, diferença de $0,63 \mathrm{~mm}$ ou $0,63 \mathrm{l} / \mathrm{m}^{2}$. O mesmo ocorre para a vazão de pico, que, para o ano de $1980, \mathrm{td}=20$ minutos ( 0,33 horas), apresentou uma vazão de $4,08 \mathrm{~m}^{2} / \mathrm{s}$, que passou para $9,41 \mathrm{~m}^{3} / \mathrm{s}$ no ano de 2008 (acréscimo de $\left.5,33 \mathrm{~m}^{2} / \mathrm{s}\right)$.

\section{CONCLUSÕES}

- A bacia hidrográfica do rio Ligeiro sofreu um processo de urbanização entre os períodos de 1980 e 2008. A partir de 1980 verifica-se o acelerado adensamento das áreas edificadas, com a cidade já apresentando um núcleo central com edificações muito densas e pouca superfície livre.

- O método racional apresenta facilidades de cálculo, mas superestimou as vazões máximas, comprovando ser indicado apenas para pequenas bacias hidrográficas. O método de Ven Te Chow apresentou valores mais coerentes quando comparados aos valores obtidos pelo método racional. Em bacias com maior área, como é o caso em estudo, vazões máximas podem advir de precipitação mais intensa e consequente formação do escoamento superficial, devido à variabilidade espacial da precipitação e cobertura do solo.

- A metodologia da curva número permite avaliar os impactos do uso do solo na resposta hidrológica da bacia. A aplicação dessa ferramenta está na obtenção da vazão máxima ou vazão pico, que pode ser utilizada tanto nos estudos das enchentes como também em avaliação, dimensionamento e planejamento de futuras obras de proteção contra inundações nas bacias urbanizadas, como é o caso da bacia do rio Ligeiro.

- O aumento das áreas urbanizadas refletiu diretamente na elevação das vazões de pico. Nas condições de umidade normal $\left(\mathrm{N}_{\mathrm{II}}\right)$, no tempo de recorrência de 10 anos, a vazão de pico (chuva $0,3 \mathrm{~h}$ ) elevouse de $59,76 \mathrm{~m}^{3}$ em 1980 para 72,13 $\mathrm{m}^{3}$ em 2008. Essa vazão é consequência da impermeabilização da bacia no período e da redução da área de cultivos agrícolas que, consequentemente, reduziram as taxas médias de infiltração da bacia (as áreas cultivadas em 1980 atingiam 70,63\% do total da bacia, e em 2008 foram reduzidas a um percentual de 51,98\%).

\section{REFERÊNCIAS}

BAUNGARTEN, A. P.; FELIX, D.; MÜLER, M. Determinação da precipitação efetiva na bacia do rio Chopim através do método da curva número do SCS. In: SIMPÓSIO BRASILEIRO DE RECURSOS HÍDRICOS, 15., 2003, Curitiba. Anais do... Curitiba: ABRH, 2003. CD-ROM.

CHOW, V. T.; MAIDMENT, D. R.; MAYS, L. W. Hidrologia aplicada. Santafé de Bogotá, Colombia: McGrawHill, 1994. 584 p.

INSTITUTO DE TERRAS, CARTOGRAFIA E GEOCIÊNCIAS DO PARANÁ (ITCG). Carta topográfica de Mariópolis. Paraná: 1982. 1 mapa, color., 79 cm x 95 cm. Escala 1:100.000.

Fotos aéreas do município de Pato Branco. Paraná, 1980. 36 fotografias aéreas, preto e branco. Escala 1:25.000.

INSTITUTO DE PESQUISA E PLANEJAMENTO DE PATO BRANCO (IPPUPB). Mapas de leituras técnicas. Disponível em: <www.ippupb.org.br>. Acesso em: 16/11/2008.

LANÇA, R. M. M.; RODRIGUES, A. C. Apresentação de um modelo distribuído de precipitação/ escoamento superficial. Associação Portuguesa de Recursos Hídricos. Congresso da Água ano 2000. Disponível em: <http://62.48.187.114/snig-educ/ficheiros/Agua-Precipit/5agua-comunicacao[1].pd〉. Acesso em: $16 / 11 / 2008$.

MENDES FILHO, W. M.; VENDRAME, I. F.; CARVALHO, R. G. Utilização de sistema de informações geográficas para o mapeamento do potencial de retenção de águas pluviais no município de 
São José dos Campos - SP. In: SIMPÓSIO BRASILEIRO DE SENSORIAMENTO REMOTO, 13., 2007, Florianópolis. Anais... Florianópolis: INPE, 2007. p. 3453 - 3460.

NUNES, F. G.; FIORI, A. P. A utilização do método de Ven Te Chow - Soil Conservations Service (SCS) na estimativa da vazão máxima da bacia hidrográfica do rio Atuba. Revista Eletrônica Geografar, v. 1, n. 2, p. 139 - 155, 2007.

PINHEIRO, A.; BADIA, S. B. Efeitos da curva-chave sobre a curva de permanência dos escoamentos em uma bacia agrícola. Revista de Estudos Ambientais REA, Blumenau, v. 10, n. 2, p. 64 - 70, 2008.

SECRETARIA DE MEIO AMBIENTE E RECURSOS HÍDRICOS (SEMA). Bacia do rio Iguaçu: bacias hidrográficas do Paraná, uma série histórica. Curitiba: Estado do Paraná, 2000. v. 1.

SUPERINTENDÊNCIA DE DESENVOLVIMENTO DE RECURSOS HÍDRICOS E SANEAMENTO AMBIENTAL (SUDERHSA). Mapa de chuvas intensas. Disponível em: <www.suderhsa.pr.gov.br>. Acesso em: 21/01/2009.

TABALIPA, N. L. Estudo da estabilidade de vertentes da bacia do rio Ligeiro, Pato Branco, Paraná. 264 p. Tese (Doutorado em Geologia) - Universidade Federal do Paraná, Curitiba, 2008.

TUCCI, C. E. M. (org.). Hidrologia: ciência e aplicação. 2. ed. Porto Alegre: Editora da Universidade; ABRH, 1997. v. 4.

WATTS, L. F.; HAWKE, R. M. The effects of urbanization on hydrologic response: a study of two coastal catchments. Journal of Hydrology (NZ), New Zealand Hydrological Society, v. 42, n. 2, p. 125 - 143, 2003. 